\title{
Engenharia de features linguísticas para classificação de triplas relacionais
}

\author{
Elian Conceição Luz ${ }^{1,2}$, Camilla Rastely da Silva ${ }^{2}$, Daniela Barreiro Claro ${ }^{1}$ \\ ${ }^{1}$ FORMAS Research Group, Departamento de Ciência da Computação \\ Instituto de Computação - Universidade Federal da Bahia, \\ 40170-110 - Salvador, BA, Brazil \\ ${ }^{2}$ Instituto de Letras - Universidade Federal da Bahia \\ 40170-110 - Salvador, BA, Brazil \\ \{elianc, camillars, dclaro\}@ufba.br
}

\begin{abstract}
In this study, linguistic features were listed for classification of triples, based on a parallel corpus in Galician, Brazilian Portuguese (PT-BR) and European Spanish (EE). In the experiments, at the syntactic level, a relevant performance of features that offer greater difficulty to extract valid triples was observed, such as the co-related null object/subject and the verb-subject inversion, as well as relational triples that form ungrammatical sub-sentences. At the morphological level, it was observed that the grammatical class of the initial word of each sub-sentence, especially when they are prepositions, were relevant for the classification of the triples.
\end{abstract}

Resumo. Neste estudo, elencaram-se features morfossintáticas para classificação de triplas, com base em um corpus paralelo em Galego, Português do Brasil e Espanhol Europeu. Nos experimentos, a nível sintático, observou-se desempenho relevante de features que oferecem maior dificuldade para extrair triplas válidas, como as co-relacionadas a objeto/sujeito nulo e a inversão verbo-sujeito, bem como triplas relacionais que formam sub-sentenças agramaticais. A nível morfológico, observou-se que a classe gramatical do vocábulo inicial de cada sub-sentença, sobretudo quando são preposições, foram relevantes para a classificação das triplas.

\section{Introdução}

$\mathrm{O}$ exponencial crescimento do volume de documentos digitais escritos em linguagem natural impulsiona a demanda por modelos automáticos capazes de extrair informação de dados não-estruturados. Nessa perspectiva, a Extração de Informação Aberta (EIA) possibilita a estruturação da linguagem natural em triplas relacionais ao processar as sentenças de um texto, obtendo uma estrutura composta por três elementos ( $\arg 1$, relação, $\arg 2)$ [Barbosa 2018]. Por exemplo, a partir da sentença 'João ama Maria', pode-se extrair a tripla válida ('João', 'ama', 'Maria').

A motivação de compor tarefas de PLN em perspectiva multilíngua é ampliar o alcance dos métodos de EIA para fora do alcance da língua inglesa. Em relação ao Português (PT-BR) e ao Espanhol Europeu (EE), por exemplo, mesmo sendo línguas de 
ampla circulação e com números expressivos de falantes, ambas apresentam recursos escassos para automação de tarefas do PLN, o que é ainda mais evidente em relação à língua galega. Nessa perspetiva, este estudo favorece o desenvolvimento de métodos capazes de extrair informação de textos redigidos nessas três línguas. Outrossim, a descrição de características de língua propicia maior independência de domínio, contribuindo para a melhoria da Extração de Informação Aberta (EIA) e para a capacitação linguística dessas línguas [Calvet 2005][Gauger 1989].

Tradicionalmente, a EIA inclui métodos de extração de triplas que, em sua maioria, foram desenvolvidos com base em corpus de língua inglesa, considerando suas especificidades linguísticas, como a maior predominância do preenchimento do sujeito e a ordem sujeito-verbo, características que não são predominantes em línguas românicas[Barbosa 2018]. Muitos estudos propuseram a tradução das features para a língua-alvo, o que não permitiu obter resultados satisfatórios. A dificuldade aumenta quando se trata de abordagens multilínguas. Assim, o principal propósito deste trabalho foi determinar as features linguísticas por meio das triplas extraídas de corpora em línguas românicas: Galego, Português (PT-BR) e Espanhol Europeu (EE). A metodologia adotada analisou as características linguísticas genéricas por meio de uma revisão de estudos da Linguística Formal, em destaque para os estudos de base gerativista [Chomsky and Lasnik 2008] [Chomsky 2014].

Este artigo está estruturado como segue: a seção 2 apresenta a problemática do estudo e hipóteses para o direcionamento dos experimentos; a seção 3 descreve as features elencadas para o desenvolvimento dos experimentos, construídas com base na descrição linguística das línguas-alvo; na seção 4, destacam-se a constituição do corpus, os experimentos e a avaliação das features; e , por fim, a seção 5 consolida os principais resultados e finaliza o estudo realizado.

\section{Features linguísticas nas três línguas românicas}

Tradicionalmente, a Extração de Informação em documentos busca explorar padrões que expressam relações predefinidas, como geral-específico, parte-todo, localidade e pertencimento. Em sintonia com o ambiente multifacetado da Web, a Extração de Informação Aberta (EIA) não limita o tipo de relação, possibilitando a análise de texto em diferentes domínios. Uma possibilidade é a extração de triplas relacionais por meio de métodos de Processamento de Linguagem Natural (PLN), cuja estrutura composta por três elementos (arg1, relação, arg2) é capaz de representar conhecimento de forma mais flexível, a exemplo de atributos, eventos, fatos e entidades [Barbosa 2018].

No entanto, em sua maioria, os métodos de EIA ainda estão focalizados em domínios linguísticos específicos, enquanto cada vez mais a Web concentra textos de diversas línguas e domínios, evidenciando as especificidades dos métodos que dificilmente são replicáveis a contextos mais amplos. Ao considerar as línguas selecionadas para estudo, observam-se aproximações e divergências com possíveis implicações para extração e validação de triplas relacionais em perspectiva multilíngua. Para tal, faz-se necessária descrição linguística dos domínios de língua para a assertiva execução de tarefas de extração e validação de triplas relacionais. Como um exercício da dificuldade em elencar convergências entre esses domínios, pode-se tomar como exemplo as sentenças e suas respectivas extrações apresentadas logo abaixo: 


\section{Galego}

Bastas querer atopar os teus funcionarios, que [sujeito nulo] aparecen no mesmo instante. ([sujeito nulo], bastas querer atopar, os teus funcionarios)

\section{Português Brasileiro}

Basta você querer encontrar seus funcionários, que eles aparecem no mesmo instante.[Fanjul 2014]

(você, basta querer encontrar, seus funcionários)

\section{Espanhol Europeu}

Basta que quieras encontrar a tus empleados, que [sujeito nulo] aparecen en el mismo instante. [Fanjul 2014]

([sujeito nulo], basta que quieras encontrar a, tus empleados).

Em estudos anteriores, demonstrou-se a importância das preposições para análise da estrutura das sentenças. Observou-se que sentenças maiores apresentam uma maior dificuldade para as tarefas de extração, pois elas, geralmente, formam períodos mais complexos. Pode-se, também, considerar que a presença de determinada classe gramatical é mais frequente em cada elemento da tripla, como são os nomes no argumento 1 e no argumento 2, por outro lado, os verbos flexionados são necessários na relacional entre os argumentos.

Por fim, as classes gramaticais das palavras em posição final e inicial indicam características importantes, como pode ser observado na sétima feature do Reverb [Fader et al. 2011]. Por exemplo, o conjunto de features proposto no modelo indica a transitividade verbal: se é direta ou indireta. Outrossim, pode ser indicativa de inversão da ordem frasal, bem como deslocamento com formação de tópico, com o deslocamento do complemento adverbial de lugar. Neste caso, é possível identificar o deslocamento pela posição da preposição 'em' no início da sentença.

\section{Engenharia de Features}

A partir da revisão bibliográfica de features propostas de trabalhos relacionados, selecionaram-se algumas características possivelmente relevantes para classificação de triplas relacionais no Galego, Português Brasileiro e Espanhol Europeu. Fez-se necessário conhecimento de domínio para análise assertiva das características de língua e correta aplicação das técnicas de análise de dados. O objetivo foi extrair o máximo de informações relevantes para análise, o que foi consolidado nas features elencadas na Tabela 1.

Ao observar as features, destaca-se, por exemplo, que as preposições ajudam a identificar a função sintática dos itens de uma sentença.

\section{Exemplo 1}

(1) Na segunda, João foi à escola.

A presença de tópico está relacionada a outros fenômenos sintáticos, como o sujeito nulo [de Castilho et al. 1973] [Chomsky and Lasnik 2008].

\section{Exemplo 2}


Table 1. Features propostas neste estudo

\begin{tabular}{c|l}
$N$ & Feature \\
\hline \hline 1 & O Arg1 não é vazio e está contido na sentença \\
2 & O Arg2 não é vazio e está contido na sentença \\
3 & A Rel está contida na sentença \\
4 & Classe gramatical da palavra no início da sentença \\
5 & Classe gramatical da palavra no início do Arg1 \\
6 & Classe gramatical da palavra no início do Arg2 \\
7 & Classe gramatical da palavra no início do Rel \\
8 & As preposições em início de sentença \\
9 & As preposições em início de Arg1 \\
10 & As preposições em início de Arg2 \\
\hline
\end{tabular}

(2) Onde está João?

(3) No domingo, [sujeito nulo] vai para escola.

No corpus estudado, é possível observar esse mesmo fenômeno em uma das sentenças nas três línguas estudadas. A partir dessa amostra, é possível observar um exemplo de como o deslocamento se relaciona com a ocorrência do sujeito nulo nessas línguas.

\section{Excerto do corpus 1}

(4) GL

Sentença:Na política interna [sujeito nulo] mostrouse sempre a favor dun goberno de conciliación nacional e foi contrario á unificación de Moldávia coa Romanía.

Tripla válida: ([sujeito nulo], foi contrario á, unificación de Moldávia coa Romanía)

(5) $\mathbf{E E}$

Sentença: En política interior [sujeito nulo] se mostró siempre a favor de un gobierno de conciliación nacional y fue contrario a la unificación de Moldavia con Romanía. Romanía)

Tripla válida: ([sujeito nulo], fue contrario a, la unificación de Moldavia con

\section{(6) PT-BR}

Sentença: Na politica interna, [sujeito nulo] mostrou-se sempre a favor de um governo de conciliação nacional e foi contrário à unificação da Moldávia com a România. România)

Tripla válida: ([sujeito nulo], foi contrário à, unificação da Moldávia com a

Nesse ponto, observa-se que há uma relação entre o deslocamento, que pode ser identificado com as features 4 e 8 . Posto que a presença de um determinante ou nome em início de sentença indica que não houve deslocamento, enquanto a presença da preposição indica que há esse deslocamento, o que está correlacionado com a aparição do sujeito 
nulo, que se manifesta no esvaziamento do Arg 1, que pode ser identificado na feature 1 [de Castilho et al. 1973] [Chomsky and Lasnik 2008].

O fato dos argumentos (Arg1 e Arg2) e a relação (Rel) estarem ou não na sentença apresenta indício do processamento utilizado para a extração de triplas, principalmente por meio dos métodos baseados em regras. Enquanto os casos em que o Arg2 estavam vazios apresentam-se como inválidos em sua totalidade, o fato de a relação $(\mathrm{Rel})$ ter sido alterada na tripla indica um processo mais complexo de extração.

\section{Excerto do corpus 2}

(7) $\mathbf{G L}$

Sentença: Fonte:cronista cumpre do diego video diego el 10 Maradona prepara a lista para enfrentar a España. Coa clasificación ao Mundial, o entrenador Diego Maradona dará a coñecer este venres a lista de convocados para o partido ante España, o 14 de novembre en Madrid.

Tripla inválida: (el 10 Maradona, prepara para, enfrentar a España Coa)

Tripla válida: (el 10 Maradona, prepara, a lista)

(8) $\mathbf{E E}$

Sentença: Fuente: cronista cumple del diego video diego el 10 Maradona prepara la lista para enfrentar a España. Con la clasificación al Mundial, el entrenador Diego Maradona dará a conocer este viernes la lista de convocados para el partido ante España, el 14 de noviembre en Madrid.

Tripla inválida: (el 10 Maradona, prepara para, enfrentar a España Con)

Tripla válida: (el entrenador Diego Maradona, dará, la lista)

(9) PT-BR

Sentença: Fonte: repórter encontra vídeo de Diego, o 10 Maradona, Diego prepara a lista para enfrentar a Espanha. Com a classificação para a Copa do Mundo, o técnico Diego Maradona anunciará nesta sexta-feira a lista de convocações para a partida contra a Espanha, no dia 14 de novembro, em Madri.

Tripla inválida: (o 10 Maradona, prepara para, enfrentar a Espanha Com)

Tripla válida: (o técnico Diego Maradona, anunciará, a lista)

Por fim, observamos que para cada elemento da tripla, esperam-se determinadas classes gramaticais em posição inicial, sobretudo, no Arg 1 e na $R e l$, nas quais, respectivamente, são mais frequentes nomes ou determinantes e verbos flexionados.

\section{Excerto do corpus 3:}

\section{GL}

(10) Sentença: Cable audio/video estándar para Xbox 360: Conecta instantáneamente aos xogadores ao mundo de Xbox 360, introduciéndolles en gráficos e xogos de última xeneración utilizando conexión é de definición estándar.

Tripla inválida: (xogos de última xeneración, utilizando, conexión) 


\section{EE}

(11) Sentença: Cable audio/video estándar para Xbox 360: Conecta instantáneamente a los jugadores al mundo de Xbox 360, introduciéndoles en gráficos y juegos de última generación utilizando conexión es de definición estándar.

Tripla inválida: (juegos de última generación, utilizando, conexión)

\section{PT-BR}

(12) Sentença: Cabo de áudio / vídeo padrão do Xbox 360: Conecta instantaneamente os jogadores ao mundo do Xbox 360, apresentando-os a gráficos e jogos de ponta usando conexão de definição padrão.

Tripla inválida: (jogos de ponta, usando, conexão).

Destaca-se que por outro lado, no Arg 2, há uma maior variedade, posto que além dos nomes e determinantes, também são frequentes preposições e outras classes gramaticais. No entanto, notou-se que a presença, por exemplo, de verbos na posição inicial são mais frequentes em triplas inválidas, como pode ser visto no excerto 03: ('o 10 Maradona', 'para enfrentar a Espanha', 'prepara a lista de convocações'). Um ponto de dificuldade dessa extração é justamente a ordem que cada elemento da tripla se apresenta na sentença, mas novamente, a posição inicial da classe gramatical ou da preposição que inicia a Rel e o Arg 2 são pertinentes.

\section{Experimentos e resultados}

Essa seção descreve o corpus paralelo criado e os experimentos para validar o conjunto de features definida para as três línguas.

\subsection{Constituição do corpus paralelo em Galego, Português Brasileiro e Espanhol Europeu}

Um ponto sensível do desenvolvimento do método proposto é a constituição de corpora em Galego, Português (PT-BR) e Espanhol Europeu (EE). A criação de um corpus paralelo permitiu analisar as features genéricas. Para tanto, selecionou-se uma base em Espanhol que já fora submetida a experimentos por outros pesquisadores [Barbosa 2018] [Gamallo and Garcia 2011]. O corpus foi criado com 371 triplas em Espanhol Europeu (EE), das quais 271 inválidas e 100 válidas acompanhadas das sentenças das quais foram extraídas. O processo de tradução para o Português (PT-BR) e para o Galego foi realizado por especialistas em Português (PT-BR) e em Galego.

\subsection{Experimentos}

Antes de realizar os experimentos com os algoritmos de classificação, em préprocessamento, realizaram-se duas tarefas de PLN, a segmentação de sentença em palavras (tokenizer) e classificação morfológica (POS taggers). Logo após esses procedimentos, realizou-se a transformação das variáveis categóricas em binárias, na qual, por exemplo, a feature "classe gramatical da palavra no início da sentença" foi substituída por variáveis binárias que indicam a presença (1) ou não (0) de uma determinada classe gramatical na posição inicial das sentenças.

Três experimentos foram realizados com os seguintes modelos de classificação: Regressão Logística, Lazy Learning e Árvore de decisão a fim de comparar o desempenho 
de cada um na classificação das triplas em válidas ou inválidas. Para o Lazy Learning, antes de sua execução, executou-se um algoritmo de busca para localizar a quantidade ideal de vizinhos próximos com base na melhor precisão encontrada.

A seleção de features ocorreu de forma iterativa. Ao observar features que tratavam de um mesmo aspecto linguístico ou que não colaboram para a melhoria do modelo da classificação, realizaram-se ajustes, mantendo as que apresentavam um melhor desempenho na avaliação, sendo o resultado final apresentado na Tabela 1.

\subsection{Avaliação}

Com o intuito de avaliar as features e averiguar o desempenho das features, o corpus foi dividindo em holdlout de $70 \%$ para treino e $\% 30$ para teste. Além disso, para evitar overfitting, o modelo k-flod 10 foi utilizado. Ao tomar como base estudos realizados por outros pesquisadores, adotou-se a precisão como a medida mais adequada para quantificar os resultados das features como relevantes para a EIA. Em ordem de prioridade, o estudo considerou precisão, $\mathrm{f} 1$, recall e acurácia.

Table 2. Corpus paralelo - Galego

\begin{tabular}{|c|c|c|c|c|}
\hline Modelo & Precisão & F1 & Revocação & Acurácia \\
\hline Regressão Logística & $\mathbf{0 . 8 2 9 6 5 4 9}$ & $\mathbf{0 . 8 6 2 5 7 5 1}$ & 0.9029703 & 0.7650192 \\
\hline Árvore de Decisão & 0.7658444 & 0.8478815 & $\mathbf{0 . 9 0 8 9 4 7 6}$ & $\mathbf{0 . 7 6 5 8 4 4 4}$ \\
\hline 37 vizinhos próximos & 0.7877068 & 0.8267316 & 0.8788258 & 0.7386713 \\
\hline
\end{tabular}

Table 3. Corpus paralelo - Português do Brasil

\begin{tabular}{|c|c|c|c|c|}
\hline Modelo & Precisão & F1 & Revocação & Acurácia \\
\hline Regressão Logística & 0.7727757 & $\mathbf{0 . 8 3 3 4 1 8 3}$ & 0.913047 & $\mathbf{0 . 7 2 8 7 9 0}$ \\
\hline Árvore de decisão & $\mathbf{0 . 7 7 5 4 3 8 5}$ & 0.8297937 & 0.9042388 & 0.7270572 \\
\hline 17 vizinhos próximos & 0.7445605 & 0.8222542 & $\mathbf{0 . 9 2 8 7 6 5 6}$ & 0.7169777 \\
\hline
\end{tabular}

Table 4. Corpus paralelo - Espanhol

\begin{tabular}{|c|c|c|c|c|}
\hline Modelo & Precisão & F1 & Revocação & Acurácia \\
\hline Árvore de decisão & 0.773257 & $\mathbf{0 . 8 5 2 0 3 6}$ & $\mathbf{0 . 9 4 2 1 2 8 5}$ & 0.7185568 \\
\hline Regressão Logística & $\mathbf{0 . 8 1 4 2 8 9 6}$ & 0.8193759 & 0.8368115 & 0.7210765 \\
\hline 57 vizinhos próximos & 0.781657 & 0.8235130 & 0.8989786 & $\mathbf{0 . 7 4 7 2 5 5 4}$ \\
\hline
\end{tabular}

A base em Espanhol Europeu foi, também, submetida a teste em experimento realizado por [Barbosa 2018], contudo com um pré-processamento distinto. Nesse experimento, os resultados obtidos pelo modelo Regressão Logística pelas features propostas pelo CMULTI foram precisão 0.717 , f1 0.828 , revocação 0.982 e acurácia 0.714 e pelo ReVerb foram precisão 0.709 , f1 0.818, revocação 0.968 e acurácia 0.698. Assim, com excesso da revocação, as demais medidas de avaliação apontam para um melhor desempenho na classificação. 


\section{Conclusão}

Neste trabalho, apresentou-se uma pesquisa dedicada a levantar o maior número de features para classificação de triplas relacionais de corpora em Galego, Português Brasileiro e Espanhol Europeu. Nesse ponto, destaca-se, também, que aspectos linguísticos comuns ao Galego, Português e Espanhol podem impor maior dificuldade aos modelos de extração arquitetados para realidade da língua inglesa, pois a estrutura Sujeito- VerboObjeto (SVO) é menos frequentes nessas línguas.

Assim, as features selecionadas nesse estudo possibilitaram a análise das triplas relacionais em todos os conjuntos testados. Entre as features, destacaram as relacionadas à presença das preposições e demais classes gramaticas em posição inicial. Dessa forma, os experimentos realizados relacionam estudos da Linguística Formal à Engenharia de Features, colaborando para Extração de Informação Aberta em perspectiva multilíngua. Em novos experimentos, os estudo podem ser enriquecidos com a análise de dependência, adoção de novos modelos de classificação e um estudo aprofundado sobre as línguas abordadas.

\section{References}

Barbosa, G. C. G. (2018). Utilizando Features multi-idioma para classificação de triplas relacionais em português, inglês e espanhol. Masters thesis, Universidade Federal da Bahia, Salvador.

Calvet, L. (2005). As Politicas Linguísticas. Parabola, São Paulo.

Chomsky, N. (2014). The minimalist program. MIT press, Cambridge.

Chomsky, N. and Lasnik, H. (2008). The theory of principles and parameters. In Syntax, pages 506-569. De Gruyter Mouton.

de Castilho, A. T., Kato, M. A., and do Nascimento, M., editors (1973). Gramática do Português Culto falado no Brasil: a construção da sentença. Fondo de Cultura Económica, Cidade do México.

Fader, A., Soderland, S., and Etzioni, O. (2011). Identifying relations for open information extraction. Proceedings of the Conference on Empirical Methods in Natural Language Processing, 8(4):2011. p. 1535-1545.

Fanjul, A. P. (2014). Conhecendo assimetrias: a ocorrência de pronomes pessoais. In Syntax, pages 29-50. Parabola editorial.

Gamallo, P. and Garcia, M. (2011). Multilingual open information extraction. Portuguese Conference on Artificial Intelligence., 8(4):p. 1535-1545.

Gauger, H.-M. (1989). Introductión a la lingüistica románica. Gredos, Madrid. 